\title{
Relationships between wheeling parameters and wheelchair skills in adults and children with $\mathrm{SCl}$
}

\author{
B Sawatzky ${ }^{1,2}, \mathrm{~N}$ Hers $^{3}$ and MK MacGillivray ${ }^{2,3}$
}

Study design: Cross-sectional.

Objectives: To determine the relationships between (1) wheeling parameters using the SmartWheel Clinical Protocol (SCP) and wheelchair skills (wheelchair skills test 4.1 (WST)) and (2) push effectiveness (m per push) and the WST, among individuals with spinal cord injury.

Setting: Biomechanics Laboratory, Canada.

Methods: Sixteen adults and eight children participated in this study. Multiple regression analyses were used to determine significant SCP predictors (that is, weight-normalised peak force, speed, push frequency and mechanical effectiveness) of WST score. To determine relationships between push effectiveness and WST scores, Pearson's correlations were calculated.

Results: SCP-TILE: speed and mechanical effectiveness explained $36 \%$ of the variance in the WST SCore. SCP-RAMP and SCP-CARPET: speed explained $58 \%$ and $37 \%$ of the variance in the WST score, respectively. Push effectiveness was significantly correlated with the WST score on all three surfaces (tile, ramp and carpet).

Conclusion: Wheeling speed was a significant predictor of the WST score for all surfaces tested. Regression analyses demonstrated that SCP-RAMP had the strongest relationship with WST score. Therefore, when time is restricted, the SCP-RAMP may be the most predictive test and speed may be the most useful variable to evaluate. However, the authors do not believe that one single variable should ever replace a full assessment of skills.

Spinal Cord (2015) 53, 561-564; doi:10.1038/sc.2015.29; published online 17 February 2015

\section{INTRODUCTION}

Assessing an individual for the most appropriate wheelchair and wheelchair set-up is important for effective and safe use of the wheelchair. Appropriate assessment tools can result in a well fit and configured manual wheelchair for individuals with spinal cord injury (SCI), which could help reduce shoulder pain and improve wheelchair skills and function. The development and use of clinical tools to help evaluate wheeled mobility is essential, given that 65 million people around the world require a wheelchair for their mobility. ${ }^{1}$

Two of the most studied methods of evaluating manual wheeling capacity used by clinicians and therapists in prescribing wheelchairs are as follows: (1) the wheelchair skills test (WST) ${ }^{2}$ from Dalhousie University, which assesses specific wheelchair skills, and (2) the SmartWheel Clinical Protocol (SCP), which uses an instrumented wheel to measure kinetic and temporal-spatial variables. The WST (4.1) is a reliable measure of capacity of skill (test-retest intraclass correlation coefficient $=0.90$ ); however, it is less reliable for evaluating whether a skill is performed safely (test-retest intraclass correlation coefficients $=0.25)$. $^{3,4}$ The WST has been used by researchers as an outcome measure of total skill, as well as a clinical tool to evaluate individual skills in various adult ${ }^{4}$ and paediatric ${ }^{5}$ populations. Many studies have been published on the use of wheelchair skills assessments to evaluate an individual's capacity to propel and manoeuvre a manual wheelchair in different environmental conditions. ${ }^{6-9}$ The skills included in the WST were modelled on activities of daily living and not surprisingly have been shown to predict quality of life and community integration. ${ }^{10}$

In addition to wheelchair skills tests that have gained popularity among researchers and clinicians, instrumented measurement wheels have been used by researchers and rehabilitation centres to record and evaluate handrim kinetic and temporal-spatial data during wheeling. ${ }^{11,12}$ Commercially available instrumented wheels (for example, SmartWheel ${ }^{11}$ and Optipush ${ }^{12}$ ) have been developed with software to enable clinicians to obtain biomechanical data about how a client propels their wheelchair. The SCP evaluates biomechanical variables while a client wheels on three surfaces (tile, carpet and $5^{\circ}$ ramp). A normative clinical database was created from SCP data to describe wheelchair propulsion in adults with SCI. ${ }^{11}$

The SCP is a reliable tool for wheelchair users with SCI. ${ }^{13}$ High intersession reliability was determined for force, push frequency, push angle and speed (for a single trial) with intraclass correlation coefficients ranging from 0.70 to 0.85 . Intersession reliability increased when an average of five trials were computed. The SCP average of five trials on the $5^{\circ}$ ramp surface increased the reliability to a range of $0.97-0.98 .{ }^{13}$

Askari et al. ${ }^{14}$ created the wheelchair propulsion test (WPT) in an attempt to be more inclusive of all wheelchair users including hand and foot propellers, and young children whose small diameter wheels

\footnotetext{
${ }^{1}$ Department of Orthopaedics, University of British Columbia, Vancouver, BC, Canada; ${ }^{2}$ International Collaboration on Repair Discoveries (ICORD), Vancouver, BC, Canada and ${ }^{3}$ Graduate Program in Rehabilitation Sciences, University of British Columbia, Vancouver, BC, Canada 
may not be compatible with an instrumented wheel. The WPT was created to be a 'low tech' measurement tool, which could be used in less-resourced settings as it only requires a tape measure and stopwatch to calculate speed $\left(\mathrm{m} \mathrm{s}^{-1}\right)$, push frequency (\#pushes $\mathrm{s}^{-1}$ ) and push effectiveness ( $\mathrm{m}$ per push) over $10 \mathrm{~m}$ of wheeling. This tool has a high inter-rater reliability (intraclass correlation coefficients $=0.96$, 0.83 and 0.80 for speed, push frequency and push effectiveness, respectively). However, intersession (that is, test-retest) reliability for the WPT has not yet been determined. The authors compared the WPT to an instrumented wheel and found that speed and push frequency, measured with a tape measure and stop-watch, correlated well with the values obtained from their instrumented wheel. Interestingly, push effectiveness was significantly lower when evaluated with the stop-watch method compared with the values obtained with an instrumented wheel, thus an instrumented wheel may be more accurate. ${ }^{14}$

Although there are reliable and valid tools for evaluating wheelchair propulsion, it is unclear whether they are related to each other. The primary objective of this study was to examine the relationship between the SCP variables (weight-normalised peak force, speed, push frequency and mechanical effectiveness) and the WST score among a population of adults and children with SCI. The secondary objective was to examine the relationship between the WST score and the WPT's push effectiveness in this same population. We hypothesised that SCP variables would be predictive of overall skill and specifically that the WST score and push effectiveness would be highly correlated.

\section{MATERIALS AND METHODS}

\section{Participants}

Individuals who had an SCI (traumatic/non-traumatic) and used a manua wheelchair for $>50 \%$ of their daily mobility were recruited to participate in

Table 1 Participant demographics $(n=24)$

\begin{tabular}{|c|c|c|c|c|c|}
\hline Age & Sex & Diagnosis/lesion level & Weight (kg) & Height $(\mathrm{cm})$ & Type of wheelchair \\
\hline 10 & $\mathrm{~F}$ & Spina bifida & 31 & 158 & Colours \\
\hline 11 & M & $\mathrm{T} 2 / 3$ & 61 & 166 & Quickie GT \\
\hline 12 & M & $\mathrm{SCl}$ (lesion unknown) & 79 & 165 & Quickie Q7 \\
\hline 13 & M & $\mathrm{T} 10$ & 27 & 152 & Quickie zippie \\
\hline 15 & M & $\mathrm{T} 4 / 5$ & 60 & 168 & Crossfire invacare \\
\hline 17 & M & Spina Bifida & 71 & 182 & $\mathrm{Ti}$ \\
\hline 17 & $\mathrm{~F}$ & SCI (lesion unknown) & 32 & 165 & Ti lite YG \\
\hline 17 & $\mathrm{~F}$ & Spina bifida & 64 & 157 & Invacare \\
\hline 19 & M & Spina bifida & 61 & 168 & Ti lite \\
\hline 20 & M & $\mathrm{SCl}$ (lesion unknown) & 112 & 180 & Ti lite \\
\hline 23 & $\mathrm{~F}$ & L1 & 45 & 165 & $2 \mathrm{RA}$ \\
\hline 23 & $\mathrm{~F}$ & T12 & 73 & 163 & Quickie GTA \\
\hline 25 & M & $\mathrm{T} 12$ & 60 & 166 & Ti lite TRC \\
\hline 27 & M & T2 & 79 & 191 & Quickie GT \\
\hline 30 & M & Spina bifida & 45 & 163 & Invacare \\
\hline 30 & M & $\mathrm{SCl}$ (lesion unknown) & 72 & 173 & Elevation \\
\hline 34 & M & $\mathrm{C} 4 / 5$ & 66 & 178 & Top end \\
\hline 34 & M & C5/6 (incomplete) & 72 & 180 & Elevation \\
\hline 35 & M & T12 & 62 & 175 & Quickie GT \\
\hline 35 & M & $\mathrm{T} 5 / 6$ & 88 & 178 & Quickie terminator \\
\hline 35 & M & C6 (complete) & 82 & 185 & Ti lite arrow Z \\
\hline 42 & $\mathrm{~F}$ & T5 & 68 & 165 & Invacare A4 \\
\hline 52 & $\mathrm{~F}$ & C4/5 (complete) & 57 & 173 & Quickie TI \\
\hline 55 & M & T7 & 68 & 185 & Ti lite folder \\
\hline 61 & M & $\mathrm{T} 11$ & 104 & 178 & Quickie 2 \\
\hline
\end{tabular}

Abbreviations: $\mathrm{F}$, female; $\mathrm{M}$, male; $\mathrm{SCl}$, spinal cord injury this study. Participants were recruited via advertisements at adult and paediatric outpatient rehabilitation centres. Inclusion criteria included the following: 10-65 years of age, able to understand English, follow directions and have a wheelchair with $22^{\prime}, 24^{\prime}$ or $25^{\prime}$ wheels with quick release axles. We certify that all applicable institutional and governmental regulations concerning ethical use of human volunteers were followed during the course of the study. Demographic and clinical background information were obtained from participants. This information included age, sex, diagnosis/ level of injury, height, weight and type of wheelchair used (Table 1).

\section{Data collection-WST (4.1)}

The WST Version 4.1 is an objective test of wheelchair skills. ${ }^{4}$ The WST evaluates performance and safety for 32 skills including indoor skills (for example, forward and backward wheeling), community skills (for example, wheeling over potholes) and more advanced skills (for example, skills involving wheelies). Scoring of the WST involves calculation of a total percentage score where the numerator is the total raw score (that is, the number of skills awarded a pass) and the denominator is the number of applicable skills for both capacity and safety. To ensure safety during all skills, an additional person acting as a spotter used a spotter strap attached to the wheelchair's rear axle to prevent tips. Testing was conducted by one of the two co-investigators trained in implementing the WST. Our analysis of the WST excluded three skills, including (1) gets from ground into wheelchair, (2) ascends stairs and (3) descends stairs. These skills were not performed because of time constraints or lack of adequate spotting for safety.

\section{Data collection-SmartWheel}

The SmartWheel (OutFront, Mesa, AZ, USA) was affixed to the participant's wheelchair on the side of their dominant arm. Participants were provided up to $5 \mathrm{~min}$ to familiarise themselves with the weight of the SmartWheel. The instrumented wheel uses six strain gauges to measure threedimensional forces and moments applied to the handrim. The SCP requires participants to wheel at a self-selected speed for $10 \mathrm{~m}$ or $10 \mathrm{~s}$, whichever occurs first, on three surfaces: tile, carpet and a $5^{\circ}$ ramp. The SCP software calculated several biomechanical variables including weight-normalised peak force (\%body weight), speed $\left(\mathrm{m} \mathrm{s}^{-1}\right)$, push frequency (\#pushes $\mathrm{s}^{-1}$ ) and mechanical effectiveness (tangential force per total force). ${ }^{11}$ In addition, the WPT's push effectiveness (m per push) was determined using the SmartWheel data for distance travelled and number of pushes applied to the handrim.

\section{Statistical analysis}

All statistical analyses were performed with the statistical software R (version 3.0.2, R Foundation for Statistical Computing, Vienna, Austria). ${ }^{15}$ Multiple regression analyses were used to determine which SCP variables were predictive of the WST score, with separate analyses for each of the three surfaces (tile, carpet and ramp). Pearson's correlations were performed between push effectiveness and the WST score for each of the three surfaces. Significance was set to $P<0.05$. Independent sample $t$-tests were used to compare variables between adult and paediatric participants to ensure that they were homogeneous and could be combined into one group.

Two of the SCP variables, peak force (\%body weight) and speed $\left(\mathrm{m} \mathrm{s}^{-1}\right)$, are often highly correlated and not surprisingly demonstrated multicollinearity, with the variation inflation factor values exceeding 2.0. Therefore, we opted to exclude peak force from the variable selection phase of the model building. To develop a parsimonious model, we removed the variable(s) with the highest (nonsignificant) $P$-value(s) and compared the nested models using analysis of variance. Tolerance level for variable selection was set to $\alpha=0.1$.

\section{RESULTS}

Demographics for the 24 participants including age, sex, diagnosis/ level of lesion and wheelchair type are shown in Table 1. Kinetic, temporal-spatial wheeling variables and WST scores for both adult (18-52 years) and paediatric (10-17 years) participants showed no differences between age groups except for peak force $(\mathrm{N})$ and push 
Table 2 Wheeling parameters from the SmartWheel Clinical Protocol (tile) and wheelchair skills test scores for paediatric and adult groups

\begin{tabular}{|c|c|c|c|}
\hline Age (years) & $14.0(2.9)$ & $32.4(10.4)$ & 0.000 \\
\hline Number of pushes & $7.6(1.3)$ & $7.0(3.4)$ & 0.628 \\
\hline Push frequency push s $^{-1}$ ) & $0.95(0.15)$ & $0.91(0.12)$ & 0.456 \\
\hline Push angle $\left({ }^{\circ}\right)$ & $67.3(20.7)$ & $80.0(8.3)$ & 0.041 \\
\hline Peak force (\% body weight) & $7.7(2.5)$ & $10.2(3.8)$ & 0.117 \\
\hline Mechanical effectiveness (\%) & $72.8(17.1)$ & $72.9(14.6)$ & 0.978 \\
\hline Skill (\%) & $90.6(6.9)$ & $89.0(12.1)$ & 0.729 \\
\hline
\end{tabular}

Critical $P=0.05$.

Table 3 The relationship between SmartWheel Clinical Protocol variables and WST score $(\mathrm{df}=23)$

\begin{tabular}{llr}
\hline Multiple regression: SCP variables on tile vs WST & $\beta$ & $\mathrm{t}$-value \\
\hline & & \\
Dependent variable $=$ WST; adjusted $\mathrm{R}^{2}=0.36 ;$ RMSE $=0.084 ; P=0.003$ & 0.135 & 2.694 \\
Speed & 0.249 & 2.091
\end{tabular}

Multiple regression: SCP variables on ramp vs WST

Dependent variable $=W S T$; adjusted $\mathrm{R}^{2}=0.58 ; R M S E=0.070 ; \mathrm{P}=0.000$ Speed

0.180

0.000

Multiple regression: SCP variables on carpet vs WST

Dependent variable $=W S T ;$ adjusted $\mathrm{R}^{2}=0.37 ; R M S E=0.083 ; \mathrm{P}=0.001$

Speed 0.188

Abbreviations: df, degree of freedom; RMSE, root mean square error; SCP, SmartWheel Clinical Protocol; WST, wheelchair skills test.

Table 4 Correlations between WST score and push effectiveness $(\mathrm{df}=23)$

\begin{tabular}{lrr}
\hline Pearson's moment correlation: push effectiveness vs WST score & $r$ & t-value \\
\hline Tile & 0.501 & 2.776 \\
Ramp & 0.632 & 3.914 \\
Carpet & 0.627 & 3.858 \\
\hline
\end{tabular}

Abbreviations: df, degree of freedom; WST, wheelchair skills test.

angle $\left(^{\circ}\right)$, which were significantly larger in the adult group compared to the paediatric group (Table 2). The average number of pushes from the SCP included in the multiple regression analyses was 7.1 (range 510), 8.6 (range 5-11) and 9.3 (5-10) for tile, ramp and carpet, respectively.

For the relationship between SCP-TILE and WST, speed and mechanical effectiveness explained $36 \%$ of the variance in the WST score (Table 3). For the SCP-RAMP and SCP-CARPET, speed alone explained 58 and $37 \%$ of the variance in WST score, respectively (Table 3). Push effectiveness and WST score were strongly $(r>0.5)$ correlated for all surfaces (Table 4).

\section{DISCUSSION}

This study is the first to our knowledge that explores the relationships between SCP outcomes and wheelchair skills. The primary purpose of this study was to evaluate the relationship between the SCP variables and the WST score among manual wheelchair users with SCI. Our models showed that regardless of wheeling surface, speed was a significant predictor of WST score. Pradon et al. ${ }^{16}$ also showed correlations between WST score and self-selected wheeling speed $\left(r=0.57 ; r^{2}=0.32\right)$ and maximal wheeling speed $\left(r=0.72 ; r^{2}=0.51\right)$. Although the ramp and carpet wheeling trials may not require maximal effort, they do require increased effort and power output compared with the tile trials. Wheeling on a ramp or carpet may be closer in intensity to the maximal wheeling speed test on the tile surface. If a clinician needed to minimise time spent on the SCP, using only the ramp or carpet trials could be feasible in a clinical setting and may also be predictive of a client's wheelchair skills.

The WPT may be a useful tool especially for less-resourced settings; however, the outcome's sensitivity to change is important to consider. Knowing the minimal detectable change of the WPT, specifically the push effectiveness, will be useful in determining whether it will be adopted as a valuable outcome measure for evaluating the impact of an intervention. 
Previous research has shown that wheeling biomechanics is dependent upon wheeling surface (for example, tile, ramp, and carpet). ${ }^{17}$ Koontz et al. ${ }^{17}$ found that when wheeling up a ramp, wheelchair users travelled shorter distances per push, used more pushes and had a slower average velocity, which they postulated to be the result of increased deceleration between pushes. Thus, it must be acknowledged that wheeling biomechanics performed on ramp or carpet are different from wheeling on tile.

Our study is one of the first to include adult and paediatric populations in the same cohort. Research in this field is often plagued with small sample sizes, and this study is no exception. However, we believe that the combined adult and paediatric data $(n=24)$ is sufficient to show relationships between the SCP and WST for individuals with SCI who are over 8 years of age. Surprisingly, this is the largest sample of data that evaluates both adults and children using the same protocol. There is not an abundance of literature on wheelchair propulsion in children. One study from the mid 1990's evaluated propulsion in 10 adults and 10 children, ${ }^{18}$ whereas two other studies in 2013 only assessed children $(n=13),{ }^{19}(n=2) .{ }^{20}$

We showed surprising similarities in wheeling parameters and skills between adult and paediatric wheelchair users with SCI (Table 2). Only peak force $(N)$ and push angle $\left({ }^{\circ}\right)$ were different between the two populations with adults using significantly greater peak force $(N)$ and longer push angles. Adults appear to use greater peak force on the basis of their larger mass considering that there were no differences in weight-normalised peak force. Our findings support the work by Bednarczyk et al. ${ }^{18}$ who concluded that kinematics and kinetics were similar in adult and paediatric populations with SCI. Our paediatric SCI population was fortunate to have ultra lightweight wheelchairs, which were generally well fit. In addition, many children had participated in wheelchair sports, which allowed them to learn higher level skills. The two youngest participants (10 and 11 years old) had the smallest push angles $\left(39.2^{\circ}\right.$ and $58^{\circ}$, respectively); however, their wheeling speeds (1.0 and $1.2 \mathrm{~m} \mathrm{~s}^{-1}$, respectively) were not different compared with the adult speeds. Despite the age differences between the two populations evaluated in this study, the data support combining these two groups for the purpose of our analyses (Table 2). Although we standardized tyre pressure by pumping participants' tyres to the maximum recommended pressure, there may be confounding variables that we did not control for, such as wheelchair training experience, self-efficacy, level of SCI and wheelchair type or maintenance. ${ }^{21}$ Four participants (one paediatric) received $100 \%$ on the WST, which may have introduced a ceiling effect into our results; however, there were no violations of any of the model assumptions that would bring into question the validity of our results. The ceiling effect was partially due to our exclusion of two very difficult skills (ascending and descending stairs). Future studies should evaluate all skills from the WST in order to reduce this ceiling effect and incorporate the newest version of the WST (4.2). ${ }^{2}$

In conclusion, there appear to be predictive relationships between wheeling parameters and wheelchair skills for individuals with SCI. Speed on tile and carpet and speed and mechanical effectiveness on ramp were significantly predictive of the WST score. Push effectiveness was also highly correlated $(r>0.5)$ with WST score and may be a valuable outcome if skills testing is not available. Depending on the time or financial resources available to a clinician, performing a speed test on a ramp or carpet may be a valid and reliable method to follow a patient's wheelchair propulsion progress. However, the authors believe that the evaluation of biomechanical variables cannot replace a comprehensive evaluation of skills and that each tool has value in evaluating wheelchair propulsion.

\section{DATA ARCHIVING}

There were no data to deposit.

\section{CONFLICT OF INTEREST}

The authors declare no conflict of interest.

\section{ACKNOWLEDGEMENTS}

This paper was partly funded by the Natural Sciences and Engineering Research Council of Canada (RGPIN 249489-07).

1 World Health Organization. Guidelines on the provision of manual wheelchairs in less resourced settings. 2008 (Online). Available: http://whqlibdoc.who.int/publications/ 2008/9789241547482_eng.pdf (Accessed 5 January 2015).

2 'Wheelchair Skills Program', Dalhousie University, 2004-2013 (Online). Available: http://www.wheelchairskillsprogram.ca/eng/manual.htm (Accessed 5 January 2015).

3 Lindquist N, Loudon P, Magis T, Rispin J, Kirby R, Manns P. Reliability of the performance and safety scores of the Wheelchair Skills Test Version 4.1 for manual wheelchair users. Arch Phys Med Rehabil 2010; 91: 1752-1757.

4 Best KL, Kirby RL, Smith C, MacLeod D. Wheelchair skills training for communitybased manual wheelchair users: a randomized controlled trial. Arch Phys Med Rehabil 2005; 86: 2316-2323.

5 Sawatzky B, Rushton P, Denison I, McDonald R. Wheelchair skills training programme for children: a pilot study. Aust Occup Ther J 2012; 59: 2-9.

6 Kilkens O, Dallemeijer A, de Witte L, van der Woude L, Post M. The Wheelchair Circuit: construct validity and responsiveness of a test to assess manual wheelchair mobility in persons with spinal cord injury. Arch Phys Med Rehabil 2004; 85: 424-431.

7 Routhier F, Vincent C, Desrosiers J, Nadeau S, Guerette C. Development of an obstacle assessment of wheelchair user performances (OCAWUP): a content validity study. Technol Disabil 2004; 16: 19-31.

8 Cowan R, Nash M, de Groot S, van der Woude L. Adapted manual wheelchair circuit: test-retest reliability and discriminative validity in persons with spinal cord injury. Arch Phys Med Rehabil 2011; 92: 1270-1280.

9 Kilkens O, Post M, van der Woude L, Dallmeijer A, van den Hauvel W. The wheelchair circuit: reliability of a test to assess mobility in persons with spinal cord injuries. Arch Phys Med Rehabil 2002; 83: 1783-1788.

10 Hosseini S, Oyster M, Kirby R, Harrington A, Boninger M. Manual wheelchair skills capacity predicts quality of life and community integration in persons with spinal cord injury. Arch Phys Med Rehabil 2012; 93: 2237-2243.

11 Cowan RE, Boninger ML, Sawatzky BJ, Mazoyer BD, Cooper RA. Preliminary outcomes of the SmartWheel User's Group Database: a proposed framework for clinicians to objectively evaluate manual wheelchair propulsion. Arch Phys Med Rehabil 2008; 89 260-268.

12 Kwarciak AM, Turner JT, Guo L, Richter WM. Comparing handrim biomechanics for treadmill and overground wheelchair propulsion. Spinal Cord 2011; 4: 457-462.

13 Lui J, MacGillivray M, Sawatzky B. Test-retest reliability and minimal detectable change of the SmartWheel clinical protocol. Arch Phys Med Rehabil 2012; 93: 2367-2372.

14 Askari S, Kirby RL, Parker K, Thompson K, O'Neil J. Wheelchair propulsion test: development properties of a new test for manual wheelchair users. Arch Phys Med Rehabil 2013; 94: 1690-1698.

$15 \mathrm{R}$ Development Core Team R: A language and environment for statistical computing. $\mathrm{R}$ Foundation for Statistical Computing: Vienna, Austria, 2008.

16 Pradon D, Pinsault N, Zory R, Routhier F. Could mobility performance measures be used to evaluate wheelchair skills? J Rehabil Med 2012; 44: 276-279.

17 Koontz AM, Cooper RA, Boninger ML, Yang Y, impink BG, van der Woude LHV. A kinetic analysis of manual wheelchair propulsion during start-up on select indoor and outdoor surfaces. J Rehabil Res Dev 2005; 42: 447-458.

18 Bednarczyk JH, Sanderson DJ. Limitations of kinematics in the assessment of wheelchair propulsion in adults and children with spinal cord injury. Phys Ther 1995; 75: 281-289.

19 Slavens BA, Graf A, Krzak J, Vogel L, Harris G Upper Extremity Wheelchair Kinematics in Children with Spinal Cord Injury. 33rd Annual International Conference of the IEEE EMBS Boston, Massachusetts USA, August 30 - September 3, 2011.

20 Schnorenberg A, Slavens B, Wang M, Vogel L, Smith P, Harris G. Biomechanical model for evaluation of pediatric upper extremity joint dynamics during wheelchair mobility. J Biomech 2014; 47: 269-2.

21 Phang S, Ginis K, Routhier F, Lemay V. The role of self-efficacy in the wheelchair skills-physical activity relationship among manual wheelchair users with spinal cord injury. Disabil Rehabil 2012; 34: 625-632. 